\title{
High-pressure structural change in the ferroelectric layered perovskite $\mathrm{Sr}_{2} \mathrm{Nb}_{2} \mathrm{O}_{7}$
}

\author{
Javier Alanis, ${ }^{1}$ Hiram Joazet Ojeda-Galván, ${ }^{1,2}$ M. C. Rodríguez-Aranda, ${ }^{2, *}$ A. G. Rodríguez, ${ }^{2}$ Harumi Moreno García, ${ }^{2}$ \\ Jorge Íñiguez, ${ }^{3,4}$ María Eugenia Mendoza, ${ }^{1}$ and Hugo R. Navarro-Contreras ${ }^{2, *}$ \\ ${ }^{1}$ Instituto de Física, Benemérita Universidad Autónoma de Puebla, Av. San Claudio and Boulevard 18 Sur, \\ Col. San Manuel, Ciudad Universitaria, Puebla, Pue. 72570, México \\ ${ }^{2}$ Coordinación para la Innovación y Aplicación de la Ciencia y la Tecnología (CIACYT), \\ Universidad Autónoma de San Luís Potosí, Álvaro Obregón 64, San Luis Potosí, S.L.P. 78000, México \\ ${ }^{3}$ Materials Research and Technology Department, Luxembourg Institute of Science and Technology (LIST). \\ Maison de l'innovation 5, Avenue des Hauts-Fourneaux, L-4362 Esch-sur-Alzette, Luxembourg \\ ${ }^{4}$ Physics and Materials Research Unit, University of Luxembourg, 41 Rue du Brill, L-4422 Belvaux, Luxembourg
}

(Received 28 March 2019; revised manuscript received 26 June 2019; published 26 August 2019)

\begin{abstract}
The structural changes of sintered powder samples of perovskite-slab layered polycrystalline $\mathrm{Sr}_{2} \mathrm{Nb}_{2} \mathrm{O}_{7}(\mathrm{SNO})$ ferroelectric compound subjected to high pressures are here investigated. The samples were prepared using a solid-state reaction in the presence (SNOE) or absence (SNO) of an applied electric field. Density functional theory (DFT) calculations including hydrostatic pressure indicate that SNO's ferroelectricity remains up to 25 $\mathrm{GPa}$ in the structure of space group $\mathrm{Cmc} 2_{1}$ derived from the condensation of one zone-center soft phonon. The predicted DFT theoretical structural changes are discussed and compared with the results of the experimental Raman spectra as a function of pressure. The pressure-dependent spectra were recorded from atmospheric pressure up to 11.5 and 13.5 GPa for SNOE and SNO, respectively. Both samples underwent a pressure induced phase transition from an incommensurate to a commensurate state at room temperature, $\mathrm{SNO}$ at $P_{i-c}=6.5 \pm 0.2$ $\mathrm{GPa}$, and SNOE at $P_{i-c}=6.9 \pm 0.3 \mathrm{GPa}$. The DFT calculations enable the identification of the change in phase to the orthorhombic structure with the space group $C m c 2_{1}$. The experimental values for $P_{i-c}$ are in reasonably good agreement with the theoretical predicted value of $\sim 7.3 \mathrm{GPa}$. After the critical pressures, the number of observable phonons decreases, that is, when the compound adopts a higher symmetry structure, several phonons vanish abruptly in both the SNO and SNOE samples, as expected. The Raman spectra for both samples show hysteresis effects, that is, after the pressure is removed, a few extra lines remain visible, as well as many relative intensity changes and broadenings for some phonon bands. The bulk moduli of the $\mathrm{Sr}_{2} \mathrm{Nb}_{2} \mathrm{O}_{7}$ before and after $P_{i-c}$ are calculated resulting in 117.0 and $147.8 \mathrm{GPa}$, respectively. The Grüneisen parameters of the phonons observed are finally calculated and discussed.
\end{abstract}

DOI: 10.1103/PhysRevB.100.054110

\section{INTRODUCTION}

Perovskites and perovskite-layered compounds are very interesting and attractive families of materials that exhibit a wide variety of interesting physical properties, such as piezoelectricity, ferroelectricity, magnetism, superconductivity, and multiferroic behaviors. Their properties have found many applications such as photovoltaic active compounds in solar cells [1], in perovskite electroluminescent diodes and optically pumped lasers [2], and in next-generation photodetectors [3].

For many perovskites, several of the aforementioned properties may coexist in a given compound, as they are successively revealed at different temperatures or applied pressure ranges, where the compound adopts different structural phases, which in turn provide the physical conditions that allow the manifestation of a given property.

For instance, $\mathrm{PbTiO}_{3}$ perovskite, which adopts a tetragonal phase below $13 \mathrm{GPa}$, followed by a "transition region" where

\footnotetext{
*Corresponding authors: carmen.aranda@uaslp.mx; hnavarro@ uaslp.mx
}

the crystal adopts a "close to" cubic phase, to finally adopt another tetragonal form at $18 \mathrm{GPa}$ [4], or $\mathrm{BaTiO}_{3}$, which in an analogous way has a ferroelectric tetragonal structure at zero pressure up to $2.7 \mathrm{GPa}$, where it adopts a cubic paraelectric phase, to revert to some ferroelectric undetermined lower symmetry phase above $5 \mathrm{GPa}$ [5].

Of particular interest is the phase transitions in simple perovskite compounds $A B X_{3}$ under hydrostatic pressure, which is a very active investigation area with great relevance to application-related issues. From these investigations, some general rules were formulated for the behavior of phase transitions in perovskite-type compounds under hydrostatic pressure: (a) there is a decrease in the transition temperature for perovskites that present soft zone-center distortion and octahedral rotations with small compressibility factors for the $A-X$ bond [6-8] and (b) there is an increase in the phase transition temperature for perovskites that present octahedral rotations with rigid $B X_{6}$ octahedra [6-8].

For laminar perovskites with the general formula $A_{n} B_{n} X_{3 n+2}$ ( $n$ is the number of $B X_{6}$ octahedra per layer; octahedral distortion and tilting lower the symmetry of these phases, hereafter called Brandon-Megaw compounds [9]), 


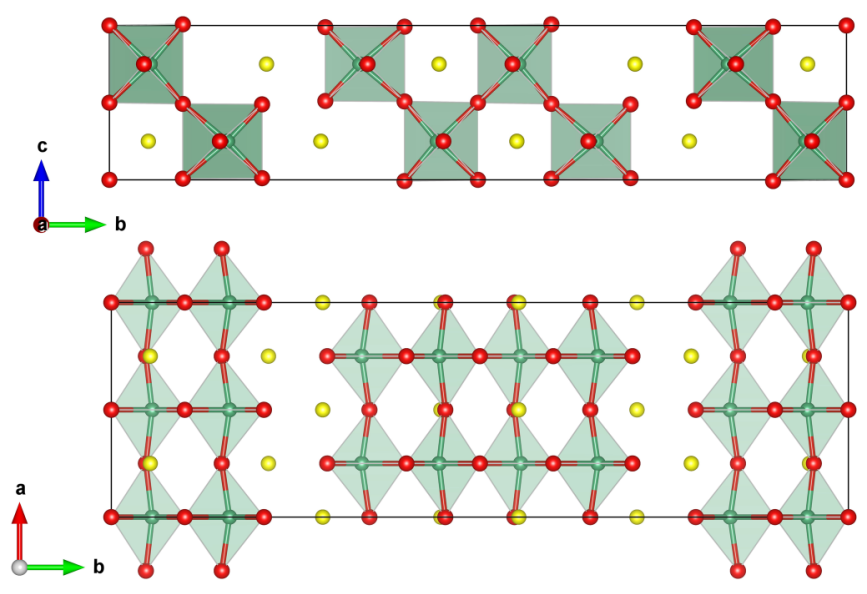

FIG. 1. An illustration of an 88-atom cell of $\mathrm{Sr}_{2} \mathrm{Nb}_{2} \mathrm{O}_{7}$ in the high symmetry PE $(\mathrm{Cmcm})$ orthorhombic cells. The yellow, green, and red balls represent $\mathrm{Sr}, \mathrm{Nb}$, and $\mathrm{O}$ atoms, respectively. The shaded polyhedrons are $\mathrm{O}_{6}$ groups.

the phase transitions under hydrostatic pressure have been studied for members of the family with $n=2$ and $X=\mathrm{F}$ [6]. Their behavior follows the second general rule for perovskites under hydrostatic pressure. In cases of other Brandon-Megaw compounds with $n>2$, only the phase transitions under hydrostatic pressure have been studied for the compounds with formula $\mathrm{La}_{\mathrm{n}} \mathrm{Ti}_{\mathrm{n}} \mathrm{O}_{3 \mathrm{n}+2}$ ( $n=4$ and 5). Two high-pressureinduced phase transitions at 8.6 and $16.7 \mathrm{GPa}$ were found for the $n=4$ compound [10] and at $18 \mathrm{GPa}$ for the $n=5$ compound [11]. All of the high-pressure phases for both compounds were not identified.

Another interesting member of the Brandon-Megaw compounds is the $n=4$ strontium (pyro) niobate $\mathrm{Sr}_{2} \mathrm{Nb}_{2} \mathrm{O}_{7}$ (Fig. 1). This compound is of interest given its ferroelectricity with high Curie temperature $\left(T_{c}=1342^{\circ} \mathrm{C}\right)$ [12], finding applications in the ferroelectric memory of field effect transistors [13], as well as for its thermoelectric properties [14] and promising photocatalytic activity [15].

$\mathrm{Sr}_{2} \mathrm{Nb}_{2} \mathrm{O}_{7}$ traverses four known phases when descending in temperature from the paraelectric phase at $T>T_{c}$. In this high temperature paraelectric phase (PE), it adopts an orthorhombic structure with space group $\operatorname{Cmcm}\left(D^{17}{ }_{2 h}\right)$ [16]. Below the paraelectric-ferroelectric transition, the orthorhombic structure adopts the space group $C m c 2_{1}\left(C^{12}{ }_{2 v}\right)$, with the main distortion (relative to the PE structure) as octahedral rotations with axis of rotation parallel to the $x$ axis, and its spontaneous polarization parallel to the $c$ axis [001] (hereafter called FE1) [17]. Below $215^{\circ} \mathrm{C}$, it undergoes a second-order phase transition with the same space group as the FE1 phase and the addition of an incommensurate state. This incommensurate state is mainly due to the displacement of the interlaminar $\mathrm{Sr}$ along the $b$ axis (hereafter called FE1-ic phase). If the incommensurate parameter is assumed to be 0 , the resultant space group will be $P b n 2_{1}$ (hereafter called FE2 phase) [16]. This new phase maintains a spontaneous polarization parallel to the $c$ axis. Below $-156^{\circ} \mathrm{C}$ a third phase transition occurs, to another incommensurate phase manifested by the appearance of an additional spontaneous polarization component along the $b$ axis (FE3) [16]. The series of phases traversed when the temperature changes are illustrated in Fig. 1 of Ref. [18].

Given the aforementioned considerations, this work presents a study of the Raman response of $\mathrm{Sr}_{2} \mathrm{Nb}_{2} \mathrm{O}_{7}$ at room temperature and subjected to increasing hydrostatic compressive pressure in the range including an atmospheric pressure of 11.5 to $13.5 \mathrm{GPa}$ for two sets of samples: the first set, grown without an applied electrical field, hereafter called the SNO sample; the second set, grown under the presence of an external applied electric field of $3.34 \mathrm{kV} / \mathrm{cm}$, perpendicular to the pellet surface, hereafter called the SNOE sample. The purpose of this electric field during the growth process is to promote a larger size and preferential orientation of the microcrystallites, as well as to influence the ferroelectric properties of the sample propitiating larger spontaneous polarizations.

\section{EXPERIMENTS AND THEORY}

\section{A. Experimental approach}

The $\mathrm{Sr}_{2} \mathrm{Nb}_{2} \mathrm{O}_{7}$ samples were prepared using a solid-state reaction of stoichiometric quantities of the oxides $\mathrm{SrO}$ (Sigma Aldrich $99.999 \%$ ) and $\mathrm{Nb}_{2} \mathrm{O}_{5}$ (Sigma Aldrich 99.99\%) by mechanical mixing and heating at $1450{ }^{\circ} \mathrm{C}$ for $7 \mathrm{~h}$. The calcinated powders were reground and uniaxially pressed into pellets at $28.5 \mathrm{MPa}$. The pellets were subsequently sintered at $1400^{\circ} \mathrm{C}$ for $2 \mathrm{~h}$. The crystal structure of the samples was characterized by $\mathrm{x}$-ray diffraction (XRD) (PANalytical Empyrean, $\lambda=1.54 \AA$, X'Celerator detector). The surface morphology of the pellets was observed using a scanning electron microscope (JSM 5400LV JEOL) as reported in Ref. [18].

Raman measurements were performed on a HORIBA XploRA PLUS micro-Raman spectrometer equipped with a single-pass monochromator and CCD detector. The spectral resolution was $1 \mathrm{~cm}^{-1}$ using the monochromator grating of 1800 grooves $/ \mathrm{mm}$. Excitation was provided by a solid-state laser $(\lambda=785 \mathrm{~nm}, 5 \mathrm{~mW})$. The spectrometer was coupled to an optical microscope Olympus BX41. The laser beam was focused on the sample using an Olympus 20X objective to a spot size $3-\mu \mathrm{m}$ diameter on the sample. The data acquisition time was $30 \mathrm{~s}$ per accumulation, averaging two co-added scans. Given the compressed granular fused morphology of all of the samples, no particular selection rules apply in these measurements. In situ, high-pressure Raman spectroscopy was performed using a Diacell CryoDAC-Mega diamond anvil cell (DAC). This cell consists of two plates attached by four screws that were hand-tightened to increase the pressure on the two diamond anvils. A metallic $(\mathrm{BeCu})$ gasket of $0.25-\mathrm{mm}$ thickness and $5-\mathrm{mm}$ diameter is placed between the anvil diamond faces. The sample was submerged in a hydrostatic fluid within a $100-\mu \mathrm{m}$ hole drilled at the gasket center and concentrically located over the face of the bottom anvil diamond. Ruby chips were also placed within the sample medium for internal pressure calibration. The hydrostatic transmission fluid consisted of a solution of 16:3:1 methanol:ethanol:water. The experimental pressure was determined from the frequency shifts in the ruby fluorescence line R1 in accordance with the equation shown by Mao et al. [19] $a=19.04 \mathrm{Mbar}, b=7.665$ (quasihydrostatic pressure), and 
$\lambda_{0}=692.22 \mathrm{~nm}:$

$$
P(\text { Mbar })=\frac{a}{b}\left\{\left[\left(1+\frac{\Delta \lambda}{\lambda_{0}}\right)^{b}\right]-1\right\},
$$

where $\Delta \lambda(\mathrm{nm})=$ ruby wavelength pressure-induced shift R1 and $\lambda_{0}(\mathrm{~nm})=$ ruby wavelength $R 1$ at atmospheric pressure.

\section{B. Theoretical approach to $\mathrm{Sr}_{2} \mathrm{Nb}_{2} \mathrm{O}_{7}$}

We used the first-principles approach as described by KingSmith and Vanderbilt to investigate displacive phase transitions applied to perovskite oxides [20]. As a starting crystalline structure, the method assumes as the initial structure a high symmetry PE phase (with intrinsic inversion symmetry) of the compound (tantamount to the equilibrium structure) and studies its stability against soft mode displacements. In this PE phase, the energy of the crystal expanded in Taylor series for the ionic displacements $x_{n}$ at second order can be written as

$$
\begin{aligned}
E^{\mathrm{dis}} & =\frac{1}{2} \sum_{m, n} K_{m n} x_{m} x_{n}=\frac{1}{2} \sum_{\alpha} k_{\alpha}\left(u_{\alpha}\right)^{2} ; \\
u_{\alpha} & =\sum_{n} x_{n} v_{\alpha n,}
\end{aligned}
$$

where $k_{\alpha}$ are the eigenvalues of the force-constant matrix $(K)$, $u_{\alpha}$ the phononic displacements, and $v_{\alpha}$ the $\alpha$-th eigenvector of $K$. The negative eigenvalues correspond to unstable structural distortions $\left(k_{\alpha}^{\text {soft }}\right)$ that could drive the structure to a phase transition. The magnitude of the instability at zero pressure is determined by the $k_{\alpha}^{\text {soft }}$ value multiplied by the squared magnitude of $u_{\alpha}^{\text {soft }}$; when an external pressure is applied, the phase transition is not guaranteed to take place due to the contributions of phonon-strain interactions (higher order terms in the Taylor series). Therefore, by adding external hydrostatic pressures, the value of the interaction terms is enhanced:

$$
E^{\mathrm{int}}=\frac{3}{3 !} \sum_{\alpha, \beta, i} C_{i \alpha \beta} \eta_{i} u_{\alpha} u_{\beta} ; C_{i \alpha \beta}=\left.\frac{\partial^{3} E}{\partial \eta_{i} \partial u_{\alpha} \partial u_{\beta}}\right|_{0},
$$

where $\eta_{i}$ is the $i$-th component of the stress tensor in the Voigt notation and $C_{i \alpha \beta}$ represents the interaction of the phononic displacement and strain.

To study $\mathrm{Sr}_{2} \mathrm{Nb}_{2} \mathrm{O}_{7}$, the software program PSEUDO [21] was used to generate the PE structure from the already reported ferroelectric structure (FE1,Cmc $\left.2_{1}\right)$ [22]. The resulting paraelectric phase for $\mathrm{Sr}_{2} \mathrm{Nb}_{2} \mathrm{O}_{7}$ has the Cmcm space group that is in accordance with the space group of isostructural $\mathrm{Sr}_{2} \mathrm{Ta}_{2} \mathrm{O}_{7}$ [23]. Also, to get indirect information about the incommensurable phase FE1-ic, we used a lower symmetry phase (FE2, $P b n 2_{1}$ ), as this phase is isostructural with the low temperature phase of $\mathrm{Ca}_{2} \mathrm{Nb}_{2} \mathrm{O}_{7}$ [24].

\section{Calculation details}

First-principles calculations were performed using density functional theory (DFT) as implemented in the Vienna $A b$ initio Simulation Package (VASP). The exchange correlation was represented within the general gradient approximation and Perdew-Burke-Ernzerhof for solids parametrization. We used the projector-augmented wave pseudopotentials to represent the ionic cores, solving explicitly for the following electrons: $\mathrm{Sr} 4 s, 4 p$, and $5 s\left(10 e^{-}\right)$; $\mathrm{Nb} 4 p, 4 d$, and $5 s\left(13 e^{-}\right)$; and $\mathrm{O} 2 s$ and $2 p\left(6 e^{-}\right)$. The electronic wave functions were represented on a plane-wave basis truncated at $500 \mathrm{eV}$. We used a $3 \times 1 \times 5$ Monkhorst-Pack $k$-point grid for Brillouin zone integrations. We worked with the 88-atom cell of SNO illustrated in Fig. 1, which is the orthorhombic FE2 $\left(P b n 2_{1}\right)$ conventional cell, double $a$ axis cell for orthorhombic FE1 $\left.(\mathrm{Cmc2})_{1}\right)$ and PE $(\mathrm{Cmcm})$ conventional cells. The calculation of the phonons was carried out using the density functional perturbation theory as implemented in VASP.

\section{RESULTS AND DISCUSSION}

\section{A. Crystal structure and microstructure}

The XRD pattern of the sample was published in the supplementary material of Ref. [18]. The position of the peaks of the pattern SNO sample match well with those of the SNO pattern in ICDD PDF04-006-6207 and no secondary phases were found. The intensity differences are the result of a preferential orientation present even in the sieve powder, where the most intense peak at $2 \theta=26.6^{\circ}$ for our SNO sample corresponded to the (080) plane. The sample had a microstructure dominated by plate-like micro-crystallites, studied and illustrated in the previously mentioned work published by our group [18], as seen in Figs. 2(a) and 2(b) in that report, where the surface normal vectors of the flakes are parallel to the direction of the $b$ axis.

\section{B. Structures of the PE (Cmcm), FE1 $\left(\mathrm{Cmc2}_{1}\right)$, and FE2 $\left(P b n 2_{1}\right)$ phases}

The results of the equilibrium structures of the studied phases are shown in Table I. The overall agreement for the phase FE1 $\left(\mathrm{Cmc2}_{1}\right)$ is acceptable, having less than $2 \%$ differences in all cell parameters between the calculated and experimental structures. For the other phases, no structural parameters have been reported.

\section{Instabilities of the PE (Cmcm), FE1 $\left(C m c 2_{1}\right)$, and FE2 $\left(P b n 2_{1}\right)$ phases}

The resultant calculated instabilities for the PE $(\mathrm{Cmcm})$ phase are presented in the form of 2 soft phonons, with (a) Cmcm soft phonon $R_{x}+D_{s r-2}$

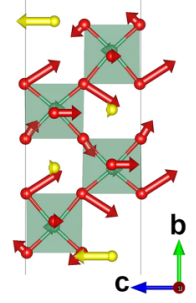

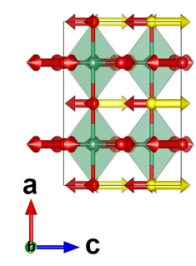

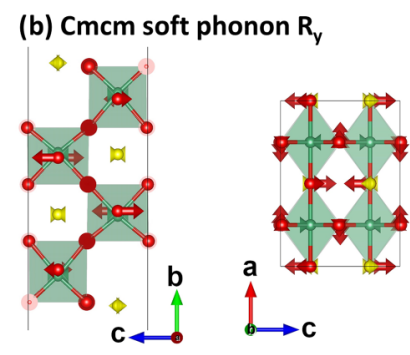

FIG. 2. Representation of the soft phonons of $\mathrm{Sr}_{2} \mathrm{Nb}_{2} \mathrm{O}_{7}$ in the PE (Cmcm) structure. (a) Soft phonon $R_{x}+D_{S r-z}$ and (b) soft phonon $R_{y}$. The yellow, green, and red balls represent $\mathrm{Sr}, \mathrm{Nb}$, and $\mathrm{O}$ atoms, respectively. The shaded polyhedrons are $\mathrm{O}_{6}$ groups and the arrows represent the respective ion displacement. 
TABLE I. Computed equilibrium structures of SNO. We show in parenthesis the experimental values reported in Ref. [24].

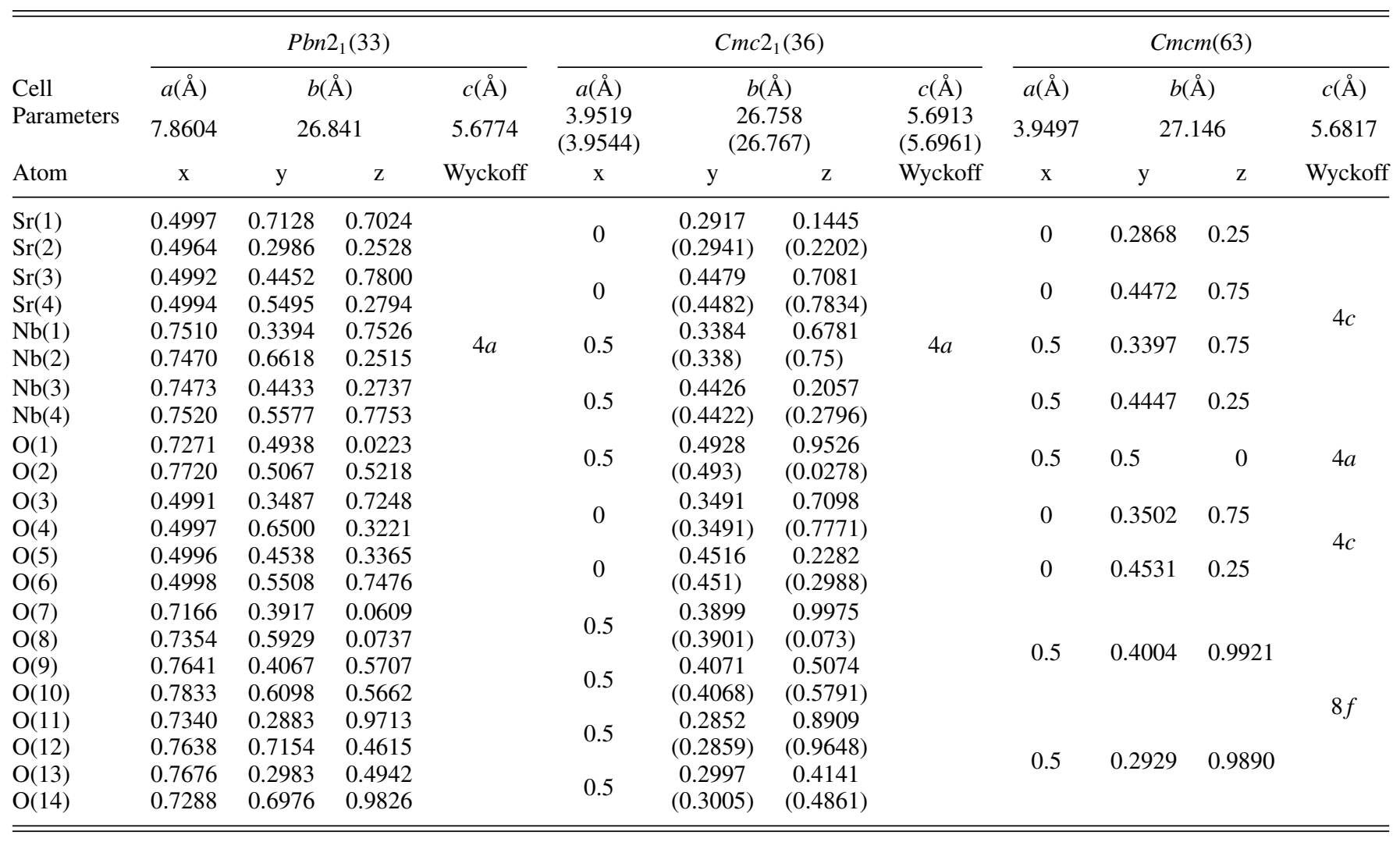

force constants of $-1.0 \mathrm{meV} / \AA^{2}\left(k_{1}^{\text {soft }}\right)$ and $-1.8 \mathrm{meV} / \AA^{2}$ $\left(k_{2}^{\text {soft }}\right)$. The $k_{1}^{\text {soft }}$ soft phonon is associated with the PE to FE1 phase transition, as shown in Fig. 2. This soft phonon can be described with two main movements, one corresponding to octahedra rotations with axis of rotation parallel to the $x$ axis (hereafter called $R_{x}$ ), and the other as displacements of the interlaminar $\mathrm{Sr}$ along the $z$ axis (hereafter called $D_{S r-z}$ ). The total soft phonon mode may be described as $R_{x}+D_{S r-z}$. The behavior of this transition is in accordance with what is observed in the isostructural material $\mathrm{La}_{2} \mathrm{Ti}_{2} \mathrm{O}_{7}$, where the condensation of two soft phonons with the same total displacements described herein are associated with the paraelectric to ferroelectric transition [25]. The $k_{2}^{\text {soft }}$ soft phonon is associated with the FE1 to FE2 phase transition, as shown in Fig. 3. This soft phonon can be described as intralaminar (a) Cmc2 1 soft phonon $R_{y}+D_{S r-y z}$
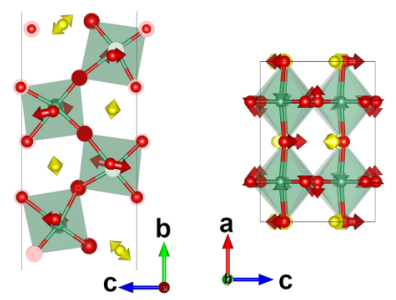

(b) Pbn2 1 soft phonon $\mathrm{D}_{\mathrm{Sr}-\mathrm{yz}}$
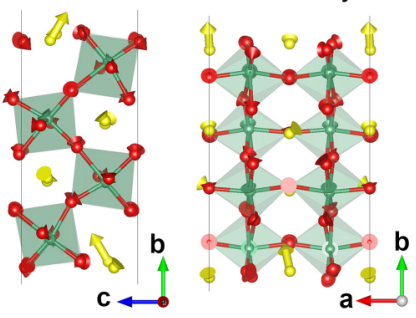

FIG. 3. Representation of the soft phonons of $\mathrm{Sr}_{2} \mathrm{Nb}_{2} \mathrm{O}_{7}$ in the a) FE1 $\left(C m c 2_{1}\right)$ structure soft phonon $R_{y}+D_{S r-y z}$ and b) FE2 $\left(P b n 2_{1}\right)$ soft phonon $\mathrm{D}_{\mathrm{Sr}-\mathrm{yz}}$. The yellow, green, and red balls represent $\mathrm{Sr}, \mathrm{Nb}$, and $\mathrm{O}$ atoms, respectively. The shaded polyhedrons are $\mathrm{O}_{6}$ groups and the arrows represent the respective ion displacement. octahedra rotations with axis of rotation parallel to the $y$ axis (hereafter called $R_{y}$ intra). We suggest that the lack of similar rotations in the interlaminar octahedra (axis of rotation parallel to the $y$ axis, $R_{y}$ inter) leads to the FE1-ic phase instead of achieving the FE2 phase. Also, as the $k_{2}^{\text {soft }}$ soft phonon has the lowest force constant value, it is to expect that this phonon condenses before the $k_{1}^{\text {soft }}$ soft phonon. In $\mathrm{Sr}_{2} \mathrm{Nb}_{2} \mathrm{O}_{7}$, the first phonon to condense (experimentally) is the $k_{1}^{\text {soft }}$ soft phonon [26]. We explain this behavior by qualitatively analyzing the rotational energy produced by the $R_{x}$ and $R_{y}$ types of displacements in a simplistic model of an ideal layered perovskite similar to ours, where we found that the rotational inertia for $R_{x}$ is approximately 2.5 times larger than $R_{y}$. This implies that for the same angle of rotation, $k_{1}^{\text {soft }}$ could represent a stronger instability than $k_{2}^{\text {soft }}$.

In the cases of the phases FE1 $\left(C m c 2_{1}\right)$ and FE2 $\left(P b n 2_{1}\right)$, we found only one soft phonon for each structure. The phase FE1 still has the instability of the $R_{y}$ soft phonon, with the addition of $\mathrm{Sr}$ displacements in the $b c$ plane $D_{S r-y z}$ (Fig. 3). The phase FE2 does not present the $R_{y}$ soft phonon and instead presents a different soft phonon that we consider related to the incommensurable part of $\mathrm{Sr}_{2} \mathrm{Nb}_{2} \mathrm{O}_{7}$ in the FE1-ic phase $\left(D_{S r-y z}\right)$. The soft phonons for the FE1 and FE2 phase indicate that the FE1-ic could be treated as an intermediated phase between them.

\section{Pressure-induced phase transition of $\mathrm{Sr}_{2} \mathrm{Nb}_{2} \mathrm{O}_{7}$}

The structural stability of $\mathrm{Sr}_{2} \mathrm{Nb}_{2} \mathrm{O}_{7}$ in the $\mathrm{PE}(\mathrm{Cmcm})$, FE1 $\left(C m c 2_{1}\right)$ and FE2 $\left(P b n 2_{1}\right)$ phases was investigated under hydrostatic pressure in the range of $0-25 \mathrm{GPa}$, and $T=0 \mathrm{~K}$. The changes in the cell parameters are shown in Fig. 4. The 

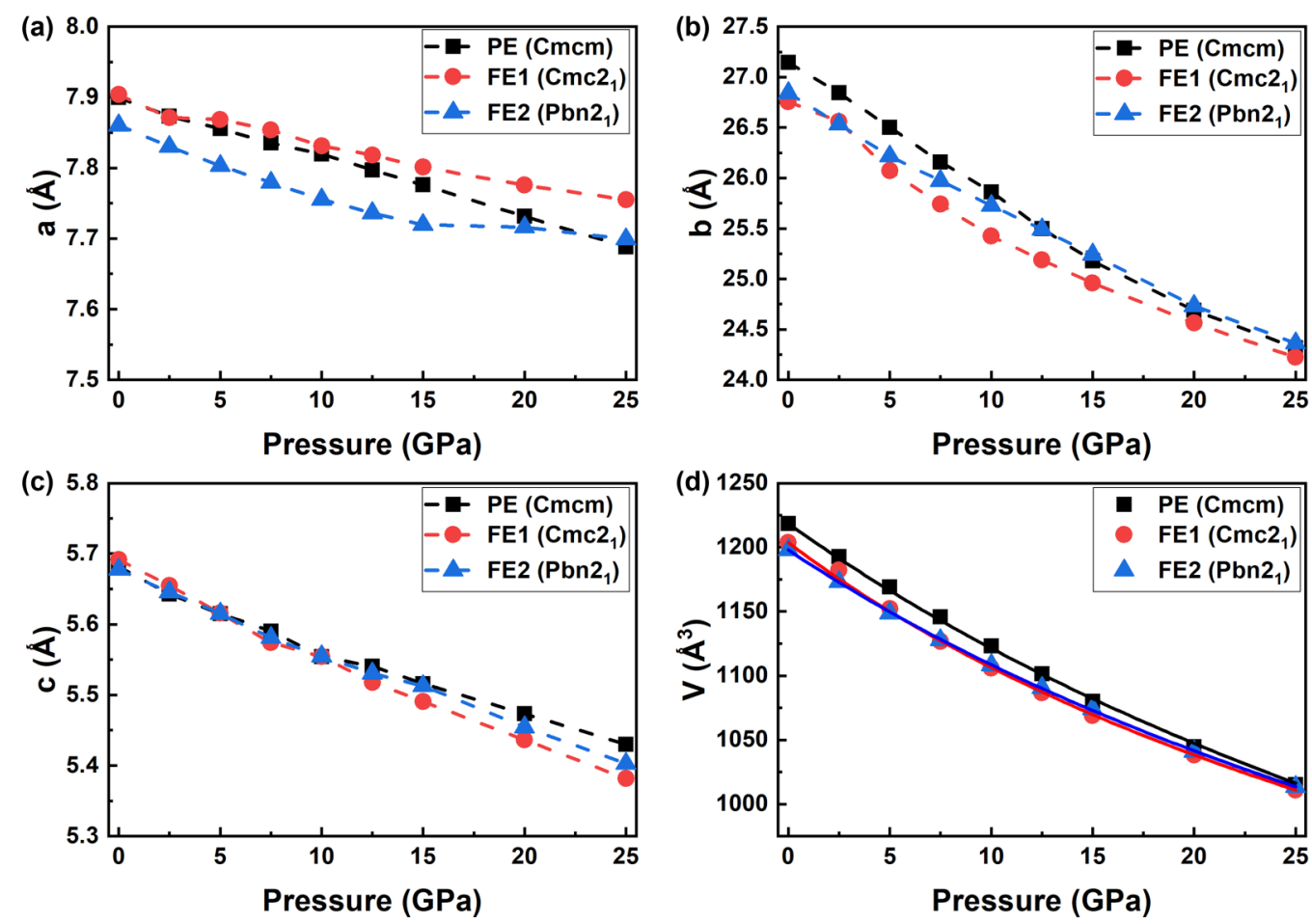

FIG. 4. Calculated cell parameters of $\mathrm{Sr}_{2} \mathrm{Nb}_{2} \mathrm{O}_{7}$ in the PE $(\mathrm{Cmcm})$, FE1 $\left(C m c 2_{1}\right)$, and FE2 $\left(P b n 2_{1}\right)$ structures analyzed in this work at $T=0 \mathrm{~K}$. The solid lines are the fits for each phase using the Murnaghan equation of state.

ratios of the relative compressibility along the $a, b$, and $c$ crystallographic axis are shown in Table II. From the volume values shown in Fig. 4(d) and using the Murnaghan relation $[10,11] V(P)=V_{0}\left[\left(B_{1} / B_{0}\right) P+1\right]^{-1 / B_{1}}$, we calculated the Bulk modulus coefficients using the linear expansion $B=$ $B_{0}+B_{1} P$ for each phase as shown in Table II. Here the $V_{0}$ value was fixed to the initial $P=0$ value in each phase. These bulk modulus values and relative compressibility along each crystallographic axis are in good agreement with those reported for other compounds of the Brandon-Megaw family $[10,11]$.

Figure 5 plots the enthalpy energy as a function of pressure. At $0 \mathrm{GPa}$, the FE2 $\left(P b n 2_{1}\right)$ phase has the lowest enthalpy, whereas at pressure above $\sim 7.3 \mathrm{GPa}$, the FE1 $\left(\mathrm{Cmc} 2_{1}\right)$ phase has the lowest enthalpy, indicating a pressure-induced phase transition from FE2 to FE1 at this value. In addition, as we consider FE1-ic as an intermediated phase between FE2 and FE1 phase, it is predicted that a pressure-induced phase transition from FE1-ic to FE1 could occur at $P \leqslant 7.3 \mathrm{GPa}$.

TABLE II. Calculated bulk modulus coefficients $B_{0}$ and $B_{1}$ and ratios of relative compressibility along the $a, b$, and $c$ crystallographic axis for the FE1, FE2, and $\mathrm{PE}_{\mathrm{Sr}_{2}} \mathrm{Nb}_{2} \mathrm{O}_{7}$ phases. Reported values for $\mathrm{La}_{n} \mathrm{Ti}_{\mathrm{n}} \mathrm{O}_{3 \mathrm{n}+2}$ are shown for qualitative comparison.

\begin{tabular}{lccc}
\hline \hline Compound/Phase & $B_{0}(\mathrm{GPa})$ & $B_{1}$ & $\Delta a / \Delta a: \Delta b / \Delta a: \Delta c / \Delta a$ \\
\hline FE2 & $114(9)$ & $3.1(8)$ & $\sim 1: 3: 1.5$ \\
FE1 & $100(8)$ & $3.9(9)$ & $\sim 1: 5.5: 3$ \\
$\mathrm{PE}$ & $108(8)$ & $2.6(8)$ & $\sim 1: 4.5: 2$ \\
$\mathrm{La}_{\mathrm{n}} \mathrm{Ti}_{\mathrm{n}} \mathrm{O}_{3 \mathrm{n}+2}(n=4)[10]$ & $121(23)$ & $18(5)$ & $\sim 1: 5: 3$ \\
$\mathrm{La}_{\mathrm{n}} \mathrm{Ti}_{\mathrm{n}} \mathrm{O}_{3 \mathrm{n}+2}(n=5)[11]$ & $142.2(11)$ & $4.3(2)$ & $\sim 1: 3: 2$ \\
\hline \hline
\end{tabular}

Similar to the PE $(\mathrm{Cmcm})$ phase at $0 \mathrm{GPa}$, we investigated the soft phonons for the same phase at a hydrostatic pressure of $10 \mathrm{GPa}$ (after the pressure-induced structural phase transition). The soft phonons $\left(k_{1}^{\text {soft }}\right.$ and $k_{2}^{\text {soft }}$ ) observed at $0 \mathrm{GPa}$ for the PE phase are still present at $10 \mathrm{GPa}$; also two other soft phonons, denoted $k_{3}^{\text {soft }}$ and $k_{4}^{\text {soft }}$, are expected at this pressure. The $k_{1}^{\text {soft }}$ soft mode at $10 \mathrm{GPa}$ has a force constant value of $-2.0 \mathrm{meV} / \AA^{2}$, where the applied pressure reduced the $\mathrm{Sr}$ displacements and maintained the octahedral rotations $\left(R_{x}\right)$,

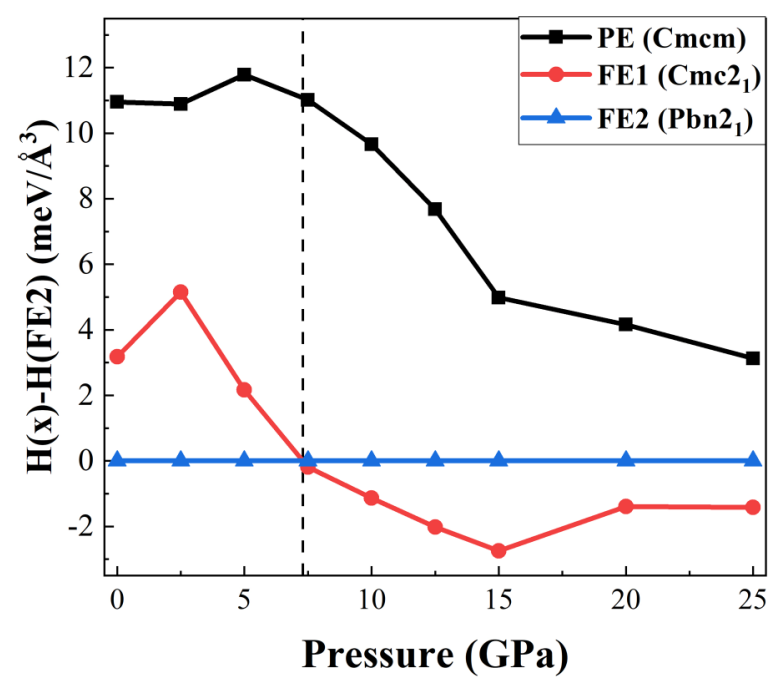

FIG. 5. Calculated enthalpy energy differences of $\mathrm{Sr}_{2} \mathrm{Nb}_{2} \mathrm{O}_{7}$ in the PE $(\mathrm{Cmcm})$, FE1 $\left(\mathrm{Cmc} 2_{1}\right)$, and FE2 $\left(\mathrm{Pbn}_{1}\right)$ phases analyzed in this work at $T=0 \mathrm{~K}$. The dashed line indicates the pressure-induced phase transition at $P=7.3 \mathrm{GPa}(\Delta H=0)$. 


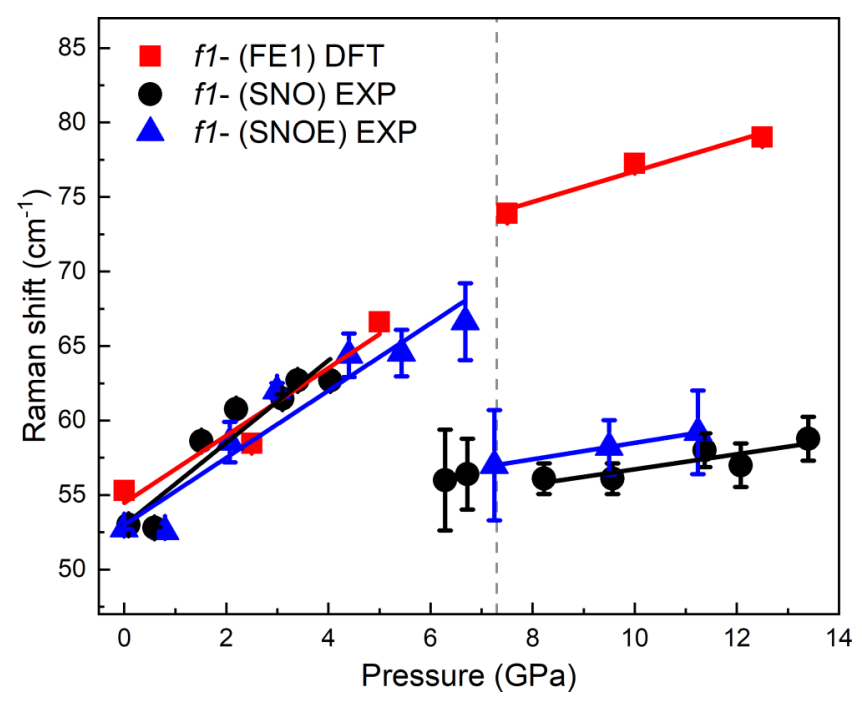

FIG. 6. Calculated frequency of the phonon $f 1$ as a function of the pressure of $\mathrm{Sr}_{2} \mathrm{Nb}_{2} \mathrm{O}_{7}$ for the FE1 $\left(C m c 2_{1}\right)$ structure analyzed in this work at $T=0 \mathrm{~K}$. The dashed line indicates the pressure-induced phase transition at $P_{i-c}=7.3 \mathrm{GPa}(\Delta H=0)$.

implying that the ferroelectricity of the material has taken place at this pressure. The $k_{2}^{\text {soft }}$ soft mode at $10 \mathrm{GPa}$ has a force constant value of $-2.5 \mathrm{meV} / \AA^{2}$, whereas the $k_{3}^{\text {soft }}$ has a force constant value of $-1.6 \mathrm{meV} / \AA^{2}$ and can be described as interlaminar octahedra rotations with axis of rotation parallel to the $y$ axis $\left(R_{y}\right.$ inter). Finally, the $k_{4}^{\text {soft }}$ has a force constant value of $-1.0 \mathrm{meV} / \AA^{2}$ and can be described as intralaminar octahedra rotations with axis of rotation parallel to the $z$ axis $\left(R_{z}\right.$-intra). This type of soft mode does not condense, possibly due to the phonon-strain interactions, in harmonic $\left(C_{i \alpha \alpha}\right)$ or anharmonic $\left(C_{i \alpha \beta}\right)$ interactions.

We also analyzed the calculated behavior of the $k_{1}^{\text {soft }}$ soft mode. As mentioned, this soft phonon is associated with the PE to FE1 phase transition. At condensation into the FE1 $\left(C m c 2_{1}\right)$ phase (hereafter called $f 1$, which corresponds to the $P$ soft mode observed in Ref. [16]) for different hydrostatic pressures (Fig. 6) the calculated slope of $f 1$ is $2.3 \pm$ $1 \mathrm{~cm}^{-1} / \mathrm{GPa}$ in the pressure range from 0 to $5 \mathrm{GPa}$; this slope changes to $1.0 \pm 0.3 \mathrm{~cm}^{-1} / \mathrm{GPa}$ in the pressure range from 7.5 to $12.5 \mathrm{GPa}$ for the FE1. When the pressure increases, the most significant change in the $f 1$ phonon is in the interlaminar $\mathrm{Sr}$, where the displacement angle changes to be parallel to the $\sim[001]$ direction. The hydrostatic pressure reduces the laminar interspace, which induces the interlaminar $\mathrm{Sr}$ to be closer to its corresponding "regular perovskite" position and the [010] direction movement is reduced.

In Fig. 6 the calculated $f 1$ phonon wave number as a function of the pressure, is presented, comparing with the $f 1$ experimental results, described in further detail in the Sec. IIIE. The calculated pressure dependences of $f 1$ are in good agreement with the experimental results. There are discrepancies in the actual calculated and observed wave number above the pressure induced transition that could be related to the fact that, in DFT calculations, we only remain in the $C m c 2_{1}$ space group when no phase transition could.
The observed wave-number difference occurring after the critical pressure (between the theoretical calculations and the experimental results shown in Fig. 6) can be attributed to the fact that for computational convenience, the $f 1$ phonon calculations are performed in a unique spatial group $\left(\mathrm{Cmc} 2_{1}\right)$. Whereas the experimental data occurring after the critical pressure $f l$ is affected by the phase transition leads to a wavenumber discontinuity.

\section{E. Experimental Raman spectra under applied pressure}

Figure 7 shows the Raman spectra observed for the compound $\mathrm{Sr}_{2} \mathrm{Nb}_{2} \mathrm{O}_{7}$ grown in the absence [Fig. 7(a)] or presence [Fig. 7(b)] of an electric field at four increasing pressures, two factors below the transition pressure.

The Raman spectra in Fig. 7 are separated into two regions (1) $50-120 \mathrm{~cm}^{-1}$ to better visualize the particular response of the two phonon lines in this region and display the pressure response of the important phonon band centered at $\sim 55 \mathrm{~cm}^{-1}$ (at RT, zero applied pressure) associated with the ferroelectric order parameter of SNO; and (2), the phonon response above $120-1000 \mathrm{~cm}^{-1}$. The dashed lines in this figure indicate the phonon lines that we were able to follow reliably with increasing pressures common to the two samples. The red line illustrates the result of the fit using Lorentzian functions to deconvolute the presence of 30 and 32 phonons in SNO and SNOE, respectively, at atmospheric pressure. The number of modes observed is lower in relation to the previous reported work (35 modes) [18]. However, three additional phonon bands were also observed $(n 1, n 2, n 3)$, which correspond to phonons reported in the work of Buixaderas et al. [26]. The number of phonons that could be identified after pressure was applied reduced to 23 . The fitting was performed using the Fityk peak fitting software version 0.9.8 [27]. The fitting was performed carefully to consider the information on the existence of the phonons our group identified at zero applied pressure (atmospheric pressure) reported in Ref. [18], but as measured inside of the diamond anvil cell.

The factor group analysis to predict the expected number of phonons observable at room temperature and zero pressure was reported by Ito et al. [28] with all of the atoms located at Wyckoff sites $4 a$ in the IC phase at room temperature, as corroborated in our $\mathrm{x}$-ray study of this compound [18]. This analysis anticipates $44 A_{1}, 22 A_{2}, 22 B_{1}$, and $44 B_{2}$ minus three acoustic modes or $132-3=129$ Raman active modes at room temperature. The observed phonon position frequencies at atmospheric pressure and their responses with increasing pressures are summarized in Table III.

With increasing pressures, most lines suffer significant broadening and decreases in intensity. These facts pose difficulties to reliably identify some of the phonon bands in the spectra under applied pressures. We could only reliably follow the evolution of 13 phonons below $P_{i-c}=6.5 \mathrm{GPa}, 11$ above this critical pressure, for $\mathrm{SNO}$, and 9 below $P_{i-c}=6.9 \mathrm{GPa}, 7$ above for SNOE.

\section{F. Raman spectra response with applied pressure}

Figure 8 presents a plot of the phonon positions identified in SNO, Fig. 8(a) and SNOE, Fig. 8(b) as a function of 
(a)

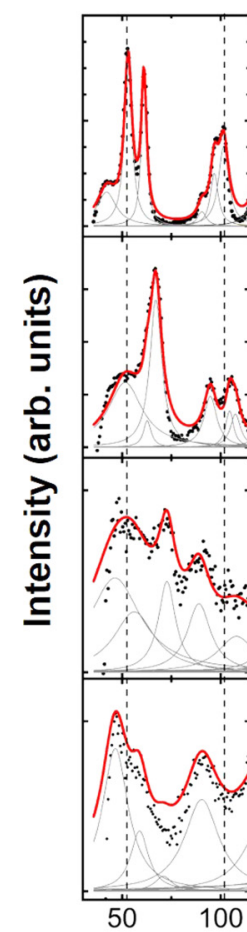

SNO

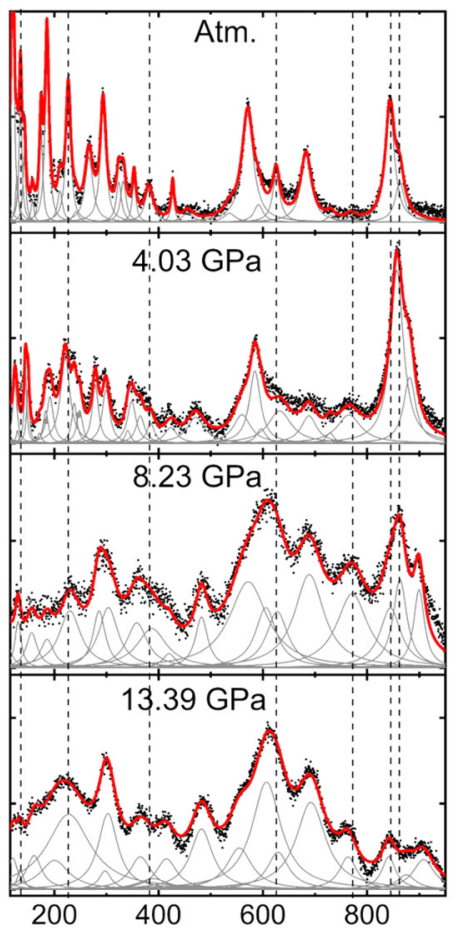

(b)

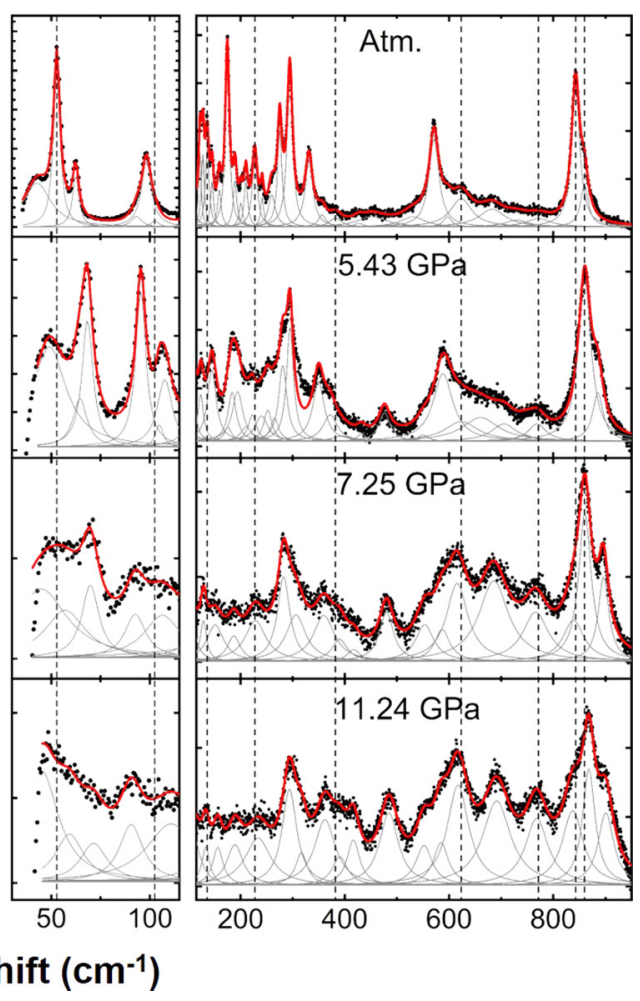

FIG. 7. Raman spectra of the perovskite layered compound $\mathrm{Sr}_{2} \mathrm{Nb}_{2} \mathrm{O}_{7}$ from atmospheric pressure to (a) $13.39 \mathrm{GPa}$ for $\mathrm{SNO}$ and (b) 11.24 for SNOE. Both spectral regions differ by a factor of two on the vertical scale, that is, the actual relative intensities of the data in the first spectral region are equivalent to multiplication by a factor of $1 / 2$ in the display. The continuous red lines represent the fit produced by $35 / 23$ identified phonons before/after pressure is applied (gray color). The dashed lines are a visual aid to appreciate the wave-number displacement of the phonon bands.

pressure. The red lines illustrate linear fittings to the pressure response for most phonons. The pressure slopes for all of the phonons observed are summarized in Table IV. Particular responses apply for the observed phonons, but for a majority, hardening is observed as the pressure increases, with only phonon $f 33$ displaying a softening response. It is evident in this figure that almost all of the phonons exhibit slope discontinuities at slightly different critical pressures for the SNO and SNOE compounds, $P_{i-c}=6.5 \pm 0.2 \mathrm{GPa}$ and $P_{i-c}=6.9 \pm 0.3 \mathrm{GPa}$, respectively. The correspondence of this critical pressure to a phase transition is strengthened by the disappearance of some of the phonons at pressures above this value, $f 9$ and $f 16$ for SNO, and $f 6$ and $f 16$ for SNOE. Thus (1) phonon $f 9$ displays contrasting behaviors among the two samples: it disappears in SNO and persists in SNOE; and (2) the dramatic change in intensity of the high wave-number phonons $f 34$ and $f 35$ (at 845.8 and $864.9 \mathrm{~cm}^{-1}$ ) for $P>P_{i-c}$; and (3) above $P_{i-c}$, a phonon called $p 4$ at $820 \mathrm{~cm}^{-1}$ appears and is persistently visible in both samples, even after the pressure is removed.

\section{G. Raman spectra after pressure is removed}

Figure 9 shows a comparison between the Raman spectra of both SNO and SNOE samples at zero pressure as pristine and after removing the pressure when the cycle of applied pressure measurements was finished. A comparison between
Figs. 9(a) and 9(b) for SNO and between Figs. 9(c) and 9(d) for SNOE clearly shows that the spectra changed after the sample was subjected to hydrostatic pressures, that is, when the relative intensities of the strongest bands are reduced and broadened and four bands appear above $300 \mathrm{~cm}^{-1}$. These are labeled $p 1, p 2, p 3$, and $p 4$ for SNO. There are only three such bands for SNOE, as $p 3$ is absent from this compound, whose positions are also summarized in Table III. Experiments are under way to characterize and ascertain the changes in either morphology or most likely in structure that may be responsible for the changes observed in the samples.

\section{DISCUSSION}

As mentioned in the theoretical results in Sec. IIID, the DFT calculations of the stability of the PE $(\mathrm{Cmcm})$, FE1 $\left(C m c 2_{1}\right)$, and FE2 $\left(P b n 2_{1}\right)$ phases investigated under hydrostatic pressure demonstrated the following results: The incommensurate phase (FE1-ic) can be considered as an intermediated structure between the FE1 to the FE2 phase and a pressure-induced structural phase transition (with the enthalpy calculations) occurs at $\sim 7.3 \mathrm{GPa}$. Our calculations are valid at room temperature due to the fact that, at this temperature the $\mathrm{Sr}_{2} \mathrm{Nb}_{2} \mathrm{O}_{7}$ adopts the FE1-ic phase.

This prediction agrees well with the experimentally observed critical pressures $P_{i-c}=6.5 \pm 0.2 \mathrm{GPa}$ and $P_{i-c}=6.9 \pm 0.3 \mathrm{GPa}$ for the SNO and SNOE compounds, 
TABLE III. Summary of phonon positions of $\mathrm{Sr}_{2} \mathrm{Nb}_{2} \mathrm{O}_{7}$ under applied pressure, below and above the critical pressure; as well as after the pressure is removed.

\begin{tabular}{|c|c|c|c|c|c|c|c|}
\hline & \multirow{2}{*}{$\begin{array}{c}\text { Ref. [18] } \\
\omega\left(\mathrm{cm}^{-1}\right) \\
\text { SNO, SNOE }\end{array}$} & \multicolumn{2}{|c|}{$\begin{array}{c}\text { Under pressure below } P_{\mathrm{c}} \\
\qquad \omega\left(\mathrm{cm}^{-1}\right)\end{array}$} & \multicolumn{2}{|c|}{$\begin{array}{l}\text { Under pressure above } P_{\mathrm{c}} \\
\omega\left(\mathrm{cm}^{-1}\right)\end{array}$} & \multicolumn{2}{|c|}{$\begin{array}{l}\text { After removing pressure } \\
\qquad \omega\left(\mathrm{cm}^{-1}\right)\end{array}$} \\
\hline & & $\mathrm{Sr}_{2} \mathrm{Nb}_{2} \mathrm{O}_{7}$ & $\mathrm{Sr}_{2} \mathrm{Nb}_{2} \mathrm{O}_{7}+\mathrm{E}$ & $\mathrm{Sr}_{2} \mathrm{Nb}_{2} \mathrm{O}_{7}$ & $\mathrm{Sr}_{2} \mathrm{Nb}_{2} \mathrm{O}_{7}+\mathrm{E}$ & $\mathrm{Sr}_{2} \mathrm{Nb}_{2} \mathrm{O}_{7}$ & $\mathrm{Sr}_{2} \mathrm{Nb}_{2} \mathrm{O}_{7}+\mathrm{E}$ \\
\hline $\mathrm{f} 1$ & 55.6 & 53.0 & 52.7 & 56.0 & 57.0 & 53.4 & 52.8 \\
\hline $\mathrm{f} 2$ & 58.3 & & & & & 60.7 & \\
\hline f3 & 64.4 & 61.0 & 62.4 & 69.8 & 69.8 & & 61.5 \\
\hline $\mathrm{f} 4$ & 66.5 & & & & & & \\
\hline n1 & & 90.5 & 93.2 & 91.7 & 92.6 & 89.5 & 89.1 \\
\hline f5 & 95.4 & 96.8 & 98.3 & 105.4 & 106.4 & 100.1 & 98.6 \\
\hline f6 & 102.5 & 101.5 & 102.6 & & & & \\
\hline $\mathrm{f} 7$ & 120.2 & 119.9 & 122.1 & 128.8 & 128.8 & 121.4 & 118.9 \\
\hline f8 & 125.5 & 128.1 & 127.6 & & & & \\
\hline f9 & 138.6 & 134.7 & 135.9 & & 151.2 & 135.0 & 132.7 \\
\hline $\mathrm{n} 2$ & & 143.0 & 143.9 & 152.2 & & & \\
\hline f10 & 148.1 & & 146.3 & & & & \\
\hline f11 & 161.1 & 160.0 & 159.2 & & & & 158.8 \\
\hline $\mathrm{f} 12$ & 177.4 & 174.3 & 174.5 & 190 & 187 & 173.1 & 179.2 \\
\hline f13 & 189.9 & 185.4 & 186.1 & & & 185.3 & 191.4 \\
\hline f14 & 203.3 & & 200.6 & & & & \\
\hline f15 & 212.9 & 212.7 & 210.3 & 228.5 & 228.5 & 209.3 & 211.2 \\
\hline f16 & 228.3 & 226.5 & 227.2 & & & 226.7 & 228.5 \\
\hline n3 & & 241.1 & 241.4 & & & 242.1 & 242.1 \\
\hline f17 & 264.6 & 266.2 & 259.8 & 280.5 & & 269.6 & \\
\hline f18 & 276.5 & & 275.0 & & 282.5 & & 273.6 \\
\hline f19 & 296.2 & 293.4 & 294.4 & 303.3 & 306.2 & 291.3 & 295.1 \\
\hline p1 & & & & & & 301.8 & 312.8 \\
\hline $\mathrm{f} 20$ & 325.8 & 323.5 & & & & 326.8 & \\
\hline $\mathrm{f} 21$ & 335.1 & 332.2 & 331.1 & 358 & 359.9 & & 340.4 \\
\hline $\mathrm{f} 22$ & 357.6 & 353.0 & 356.7 & & & 352.5 & 365.4 \\
\hline $\mathrm{f} 23$ & 378.3 & 381.4 & 382.4 & 383.3 & 390.2 & 382.37 & 382.8 \\
\hline $\mathrm{f} 24$ & 427.5 & 426.9 & 427.0 & 418.4 & 417.4 & 426.6 & 412.3 \\
\hline $\mathrm{f} 25$ & 456.0 & 456.1 & 452.8 & 481.3 & 480 & 460.4 & \\
\hline p2 & & & & & & 490.0 & 484.4 \\
\hline p3 & & & & & & 523.0 & \\
\hline f 26 & 536.9 & 558.2 & & 557.8 & 553.3 & 549.5 & 555.7 \\
\hline $\mathrm{f} 27$ & 570.1 & 572.7 & 571.2 & 592.5 & 586.8 & 573.4 & 572.9 \\
\hline $\mathrm{f} 28$ & 589 & & 596.5 & & 615.6 & & \\
\hline f29 & 633.5 & 624.4 & 624.3 & 620.5 & 685.9 & 622.1 & 613.8 \\
\hline $\mathrm{f} 30$ & 664.1 & & & & & & \\
\hline $\mathrm{f} 31$ & 686.2 & 682.9 & 684.9 & 690.8 & & 684.8 & 691.4 \\
\hline $\mathrm{f} 32$ & 731.1 & & & & & & \\
\hline f33 & 790.7 & 766.2 & 766.5 & 765.2 & 766.2 & 765.3 & 763.5 \\
\hline p4 & & & & 840.6 & 838.6 & 819.1 & 820.2 \\
\hline f34 & 845.8 & 843.7 & 842.8 & 861.8 & 860.6 & 842.4 & 844.8 \\
\hline $\mathrm{f} 35$ & 864.9 & 862.7 & 859.7 & 895.4 & 896.4 & 858.2 & 860.7 \\
\hline
\end{tabular}

respectively. Hence, we identify the crystalline structure adopted above $\sim 7 \mathrm{GPa}$ as orthorhombic belonging to the space group $C m c 2_{1}$ (FE1). As previously discussed, because $\mathrm{Sr}_{2} \mathrm{Nb}_{2} \mathrm{O}_{7}$ presents a typical perovskite behavior with hydrostatic pressure, it is expected that the already high Curie temperature should augment with the application of hydrostatic pressure, making the compound suitable for high temperature-pressure applications.

Finally, from the phonon frequency dependence with pressure, we determined the Grüneisen parameter $\gamma_{i, P}$ using the calculated bulk modulus coefficient $B_{0}$ for the FE1 phase as summarized in Table IV. This parameter $\gamma_{i, P}$ describes the variation in the vibrational frequency when a material is subjected to different pressures and is calculated using the following expression:

$$
\gamma_{i, P}=\frac{B_{0}}{\omega_{0}}\left(\frac{\partial \omega}{\partial P}\right)_{T} .
$$

The frequency-pressure slopes and the Grüneisen parameter values for the analyzed phonons are similarly summarized in Table IV. The values between 1.1 to 6.6 for $\mathrm{CaTiO}_{3}$ and -7.5 to 1.0 for $\mathrm{Pb}_{1-x} \mathrm{La}_{x} \mathrm{TiO}_{3}$ are similar to the reported experimental values for regular perovskite structures [29,30]. 
TABLE IV. Summary of the observed phonons positions inside of DAC $\boldsymbol{\omega}_{i}(\boldsymbol{P}=0 \boldsymbol{G P a})=\boldsymbol{\omega}_{i, 0}$ in $\mathrm{cm}^{-1}$ (interpolated values), wavenumber-pressure slopes $\left(\frac{\partial \omega_{i}}{\partial \boldsymbol{P}}\right)_{\boldsymbol{T}}=\boldsymbol{m}_{\boldsymbol{P}}$ in $\mathrm{cm}^{-1} / \mathrm{GPa}$ and Grüneisen pressure parameters $\boldsymbol{\gamma}_{\boldsymbol{i}, \boldsymbol{P}}$ for SNO and SNOE. Only the phonons whose response with the pressure could be reliably followed are shown.

\begin{tabular}{|c|c|c|c|c|c|c|c|c|c|c|c|c|}
\hline \multirow[b]{3}{*}{ Phonon name } & & \multicolumn{5}{|c|}{$\mathrm{SNO}\left(\mathrm{Sr}_{2} \mathrm{Nb}_{2} \mathrm{O}_{7}\right)$} & & \multicolumn{5}{|c|}{$\mathrm{SNOE}\left(\mathrm{Sr}_{2} \mathrm{Nb}_{2} \mathrm{O}_{7}+\right.$ Electric field $)$} \\
\hline & \multicolumn{3}{|c|}{$P<6.5 \pm 0.2 \mathrm{GPa}$} & \multicolumn{3}{|c|}{$P>6.5 \pm 0.2 \mathrm{GPa}$} & \multicolumn{3}{|c|}{$P<6.9 \pm 0.3 \mathrm{GPa}$} & \multicolumn{3}{|c|}{$P>6.9 \pm 0.3 \mathrm{GPa}$} \\
\hline & $\omega_{\mathrm{i}, 0}$ & $m_{\mathrm{P}}$ & $\gamma_{\mathrm{i}, \mathrm{P}}$ & $\omega_{\mathrm{i}, 0}$ & $m_{\mathrm{P}}$ & $\gamma_{\mathrm{i}, \mathrm{P}}$ & $\omega_{\mathrm{i}, 0}$ & $m_{\mathrm{P}}$ & $\gamma_{\mathrm{i}, \mathrm{P}}$ & $\omega_{\mathrm{i}, 0}$ & $m_{\mathrm{P}}$ & $\gamma_{\mathrm{i}, \mathrm{P}}$ \\
\hline f1 & 53.0 & 2.72 & 6.00 & 51.7 & 0.50 & 1.45 & 52.7 & 2.25 & 5.00 & 53.0 & 0.55 & 1.53 \\
\hline $\mathrm{f} 3$ & 61.3 & 1.31 & 2.50 & 75.4 & -0.34 & -0.53 & & & & & & \\
\hline f6 & & & & & & & 126.0 & 1.01 & 0.94 & \multicolumn{3}{|c|}{ Disappears at $\sim 7 \mathrm{GPa}$} \\
\hline f9 & 135.1 & 2.45 & 2.12 & \multicolumn{3}{|c|}{ Disappears at $4 \mathrm{GPa}$} & 135.9 & 2.13 & 1.83 & 135.9 & 2.13 & 2.32 \\
\hline $\mathrm{f} 15$ & 208.9 & 2.95 & 1.65 & 226.8 & 0.36 & 0.23 & & & & & & \\
\hline f16 & 225.0 & 2.92 & 1.52 & \multicolumn{3}{|c|}{ Disappears at $4 \mathrm{GPa}$} & 227.2 & 2.57 & 1.32 & \multicolumn{3}{|c|}{ Disappears at $\sim 7 \mathrm{GPa}$} \\
\hline f17 & 272.4 & 1.70 & 0.73 & 267.9 & 2.11 & 1.16 & & & & & & \\
\hline f19 & 293.8 & 1.71 & 0.68 & 306.4 & -0.11 & -0.05 & & & & & & \\
\hline $\mathrm{f} 23$ & 381.4 & 0.48 & 0.15 & 385.0 & 0.34 & 0.13 & 382.4 & 2.34 & 0.72 & 389.7 & 0.09 & 0.03 \\
\hline $\mathrm{f} 25$ & 456.6 & 3.95 & 1.01 & 485.9 & -0.28 & -0.08 & & & & & & \\
\hline $\mathrm{f} 27$ & 570.3 & 3.61 & 0.74 & 596.7 & 0.92 & 0.23 & & & & & & \\
\hline f29 & & & & & & & 624.3 & 7.78 & 1.46 & 676.5 & 1.35 & 0.29 \\
\hline $\mathrm{f} 33$ & 769.3 & -0.86 & -0.13 & 770.9 & -0.50 & -0.09 & 771.7 & 0.11 & 0.02 & 771.7 & 0.11 & 0.02 \\
\hline $\mathrm{p} 4$ & \multicolumn{3}{|c|}{ Appears at $\sim 6.7 \mathrm{GPa}$} & 840.9 & 0.17 & 0.03 & \multicolumn{3}{|c|}{ Appears at $\sim 7 \mathrm{GPa}$} & 842.6 & -0.47 & -0.08 \\
\hline f34 & 844.1 & 3.54 & 0.49 & 847.2 & 2.02 & 0.35 & 842.8 & 3.44 & 0.48 & 845.7 & 1.94 & 0.34 \\
\hline $\mathrm{f} 35$ & 860.8 & 5.18 & 0.70 & 879.6 & 2.34 & 0.39 & 859.7 & 5.55 & 0.76 & 888.4 & 1.11 & 0.18 \\
\hline Bulk Modulus & \multicolumn{3}{|c|}{$117.0 \mathrm{GPa}$} & \multicolumn{3}{|c|}{$147.8 \mathrm{GPa}$} & \multicolumn{3}{|c|}{$117.0 \mathrm{GPa}$} & \multicolumn{3}{|c|}{$147.8 \mathrm{GPa}$} \\
\hline
\end{tabular}

Also, we found negative values in our calculated Grüneisen parameters, which are related to phase instabilities in the octahedral rotations in the $a b$ plane, according to Peercy et al. [31], but we consider that these values are not large enough (compared to those values in Peercy et al.) to be considered. In some cases, the $\gamma_{i, P}$ changes dramatically above $P_{i-c}$, for instance, the $f 1$ phonon of SNO varies from 6.0 to 1.4. Meanwhile, for SNOE, the variation of the $f l \gamma_{1, P}$ parameter

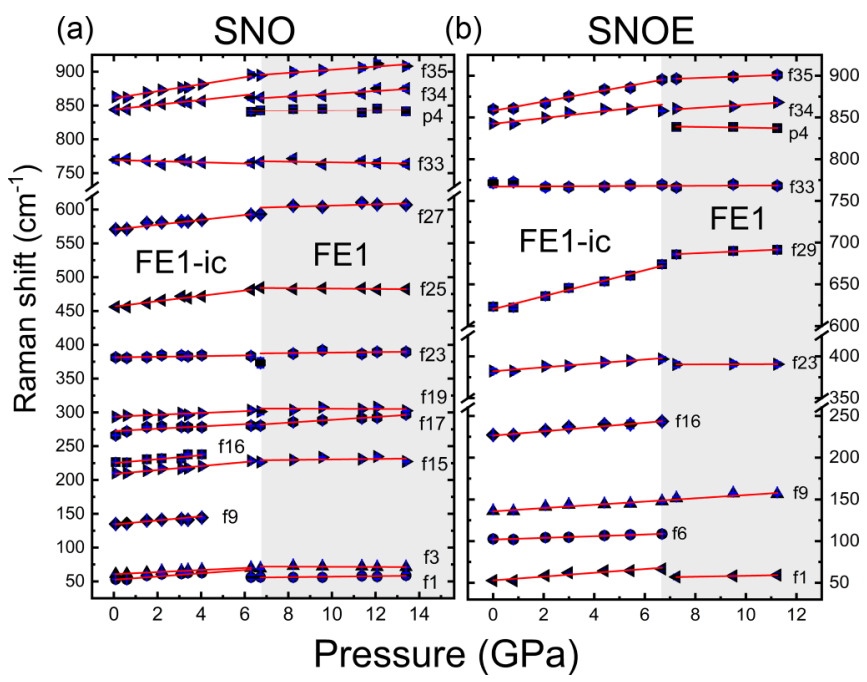

FIG. 8. Plot of the observed phonon band positions of the perovskite layered compound $\mathrm{Sr}_{2} \mathrm{Nb}_{2} \mathrm{O}_{7}$. For most of the phonons, the experimental position uncertainty (indicated with error bars for all of the other phonons) is encompassed by the symbol size. For both samples (SNO) and (SNOE), there is a critical pressure where the samples undergo a phase transition from the FE1-ic to FE1 structure as indicated by the gray shaded region.

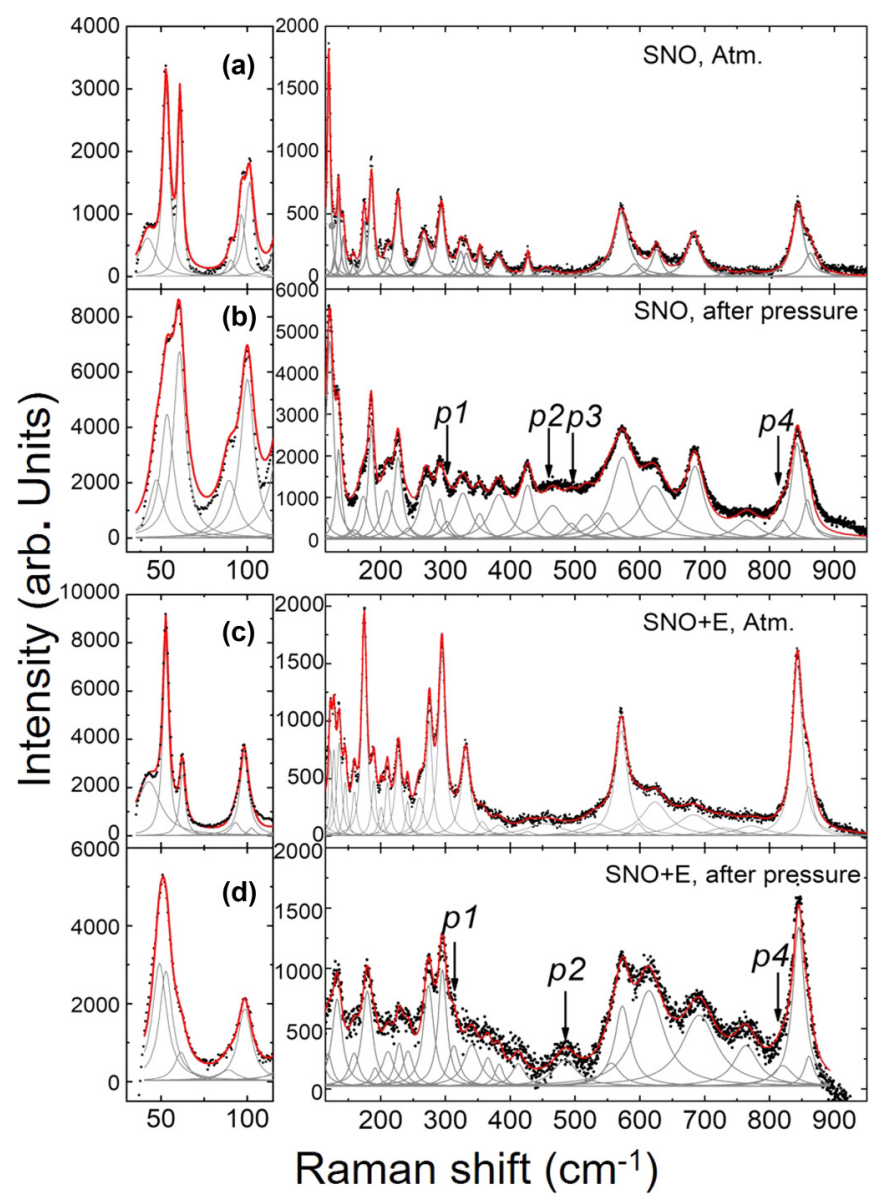

FIG. 9. Raman spectra of the perovskite layered compound $\mathrm{Sr}_{2} \mathrm{Nb}_{2} \mathrm{O}_{7}$ at atmospheric pressure before and after the samples are subjected to hydrostatic pressures. 
is smaller, from 5.0 to 1.5 . Table IV shows that the pressure dependence of $f 1$ for SNO and SNOE below $P_{i-c}$ is in good agreement with the calculated behavior for the $C m c 2_{1}$ phase; above $P_{i-c}$, SNOE remains in good agreement with the calculated behavior. In the case of SNO, $f 1$ drastically decreases this dependence with pressure. This low-dependence behavior in $f l$ can be explained with the reported effects of Ba substitution on $\mathrm{Sr}_{2} \mathrm{Nb}_{2} \mathrm{O}_{7}$ to form $\mathrm{Sr}_{2-x} \mathrm{Ba}_{x} \mathrm{Nb}_{2} \mathrm{O}_{7}$ [32], where the substitution does not significantly change the frequency of the $f 1$ phonon. Additionally, the substitution of Ba decreases the $\mathrm{NbO}_{6} R_{\mathrm{x}}$ rotations, as observed through the reduction of the spontaneous polarization [33]. Therefore, using the two mentioned phenomena with the substitution of $\mathrm{Ba}$, we can conclude that the $f l$ has a low dependence with the $\mathrm{NbO}_{6} R_{\mathrm{x}}$ rotations, which agrees with the calculated behavior mentioned in Sec. III D.

\section{CONCLUSIONS}

We studied the response of the Raman spectra of the perovskite-slab layered polycrystalline $\mathrm{Sr}_{2} \mathrm{Nb}_{2} \mathrm{O}_{7}$ ferroelectric compound to increasing hydrostatic pressures. DFT calculations enabled the identification of a phase transition at applied pressures at $P_{i-c}=6.5 \pm 0.2 \mathrm{GPa}$ and $P_{i-c}=6.9 \pm$ $0.3 \mathrm{GPa}$ for the samples grown in the absence or presence of an electric field, respectively. The theoretical results predict that the $P_{i-c}$ is expected to occur at $7.3 \mathrm{GPa}$, a value in close agreement with the experimental results. At this pressure, the sample should change from an incommensurate structure (FE1-ic) related to the space group $P b n 2_{1}$ (FE2) to an orthorhombic structure with the space group $C m c 2_{1}$ (FE1). In both phases, the calculations predict that the compound $\mathrm{Sr}_{2} \mathrm{Nb}_{2} \mathrm{O}_{7}$ remains ferroelectric. The Grüneisen parameters for the observed phonons are calculated. The variation in the value of the $f 1$ phonon following the phase transition FE1-ic to FE2 is explained through the movement of the interlaminar $\mathrm{Sr}$ for both samples. As $\mathrm{Sr}_{2} \mathrm{Nb}_{2} \mathrm{O}_{7}$ presents a typical perovskite behavior with hydrostatic pressure, it is expected that the already high Curie temperature should increase with the application of hydrostatic pressure. This makes it possible for the compound to be suitable for applications in high temperature-pressure environments.

\section{ACKNOWLEDGMENTS}

The authors acknowledge the financial support of Consejo Nacional de Ciencia y Tecnología (CONACyT) México through the projects Estancias Posdoctorales 2018(1), Ciencia Básica 256788, from FAI-UASLP, and access to Laboratorio Nacional de Análisis Físicos, Químicos y Biológicos-UASLP, during the course of this research. J.I. also acknowledges the support of the Luxembourg National Research Fund, through Grant No. FNR/P12/4853155 "COFERMAT". Furthermore, the authors gratefully acknowledge the computer resources, technical expertise, and support provided by the Laboratorio Nacional de Supercómputo del Sureste de México, CONACYT Network of National Laboratories.
[1] T. Leijtens, K. A. Bush, R. Prasanna, and M. D. McGehee, Nat. Energy 3, 828 (2018).

[2] Brandon R. Sutherland and Edward H. Sargent, Nat. Photonics 10, 295 (2016).

[3] F. Pelayo García de Arquer, A. Armin, P. Meredith, and E. H. Sargent, Nat. Rev. Mater. 2, 16100 (2017).

[4] P. E. Janolin, P. Bouvier, J. Kreisel, P. A. Thomas, I. A. Kornev, L. Bellaiche, W. Crichton, M. Hanfland, and B. Dkhil, Phys. Rev. Lett. 101, 237601 (2008).

[5] U. D. Venkateswaran, V. M. Naik, and R. Naik, Phys. Rev. B 58, 14256 (1998).

[6] T. Tohei, A. Kuwabara, T. Yamamoto, F. Oba, and I. Tanaka, Phys. Rev. Lett. 94, 035502 (2005).

[7] R. J. Angel, J. Zhao, and N. L. Ross, Phys. Rev. Lett. 95, 025503 (2005).

[8] M. Guennou, P. Bouvier, J. Kreisel, and D. Machon, Phys. Rev. B 81, 054115 (2010).

[9] F. Lichtenberg, A. Herrnberger, K. Wiedenmann, and J. Mannhart, Prog. Solid State Chem. 29, 1 (2001).

[10] F. X. Zhang, J. Lian, U. Becker, R. C. Ewing, L. M. Wang, Jingzhu Hu, and S. K. Saxena, Solid State Chem.180, 571 (2007).

[11] I. Loa, K. Syassen, X. Wang, F. Lichtenberg, M. Hanfland, and C. A. Kuntscher, Phys. Rev. B. 69, 224105 (2004).
[12] T. Williams, F. Lichtenberg, D. Widmer, J. G. Bednorz, and A. Reller, J. Solid State Chem. 103, 375 (1993).

[13] Y. Fujimori, N. Izumi, T. Nakamura, and A. Kamisawa., Jpn. J. Appl. Phys. 37, 5207 (1998).

[14] A. Sakai, T. Kanno, K. Takahashi, Y. Yamada, and H. Adachi, J. Appl. Phys. 108, 103706 (2010).

[15] A. Kudo, H. Kato, and S. Nakagawa, J. Phys. Chem. B 104, 571 (2000).

[16] K. Ohi and S. Kojima, Jpn. J. Appl. Phys. 24, 817 (1985).

[17] S. Nanamatsu, M. Kimura, K. Doi, and M. Takahashi, J. Phys. Soc. Jpn. 30, 300 (1971).

[18] J. Alanis, M. C. Rodríguez-Aranda, Á. G. Rodríguez-Vázquez, H. Joazet Ojeda-Galván, M. E. Mendoza, and H. R. NavarroContreras, J. Raman Spectrosc. 50, 102 (2019).

[19] H. K. Mao, J. Xu, and P. M. Bell, JGR Solid Earth 91, 4673 (1986).

[20] R. D. King-Smith and D. Vanderbilt, Phys. Rev. B 49, 5828 (1994).

[21] C. Capillas, E. S. Tasci, G. de la Flor, D. Orobengoa, J. M. Perez-Mato, and M. I. Aroyo, Z. Kristallogr. 226, 186 (2011).

[22] P. Daniels, R. Tamazyan, C. A. Kuntscher, M. Dressel, F. Lichtenberg, and S. van Smaalen, Acta Crystallogr. Sect. B 58, 970 (2002).

[23] N. Yamamoto, K. Yagi, G. Honjo, M. Kimura, and T. Kawamura, J. Phys. Soc. Jpn. 48, 185 (1980). 
[24] N. Ishizawa, F. Marumo, S. Iwai, M. Kimura, and T. Kawamura, Acta Crystallogr. Sect. B 36, 763 (1980).

[25] J. López-Pérez and J. Íñiguez, Phys. Rev. B. 84, 075121 (2011).

[26] E. Buixaderas, S. Kamba, and J. Petzelt, J. Phys.: Condens. Matter 13, 2823 (2001).

[27] M. Wojdyr, J. Appl. Crystallogr. 43, 1126 (2010).

[28] K. Ito, T. Horokoshi, and K. Ohi. Ferroelectrics 203, 201 (1997).
[29] P. Gillet, F. Guyot, G. D. Price, B. Tournerie, and A. Le Cleach, Phys. Chem. Minerals 20, 159 (1993).

[30] M. R. Joya, J. Barba-Ortega, and P. S. Pizani, J. Appl. Phys. 113, 013512 (2013).

[31] P. S. Peercy and B. Morosin, Phys. Rev. B 7, 2779 (1973).

[32] G. Shabbir, Y. Akishige, and S. Kojima, Sci. Technol. Adv. Mater. 6, 656 (2005).

[33] Z. Gao, H. Ning, C. Chen, R. Wilson, B. Shi, H. Ye, H. Yan, and M. J. Reece, J. Am. Ceram. Soc. 96, 1163 (2013). 